\title{
SCALE ANALYSIS OF THE SIGNIFICANCE OF DISPERSION IN MIXING-TRANSPORT IN CONDUITS
}

\author{
Guangquan Li, Yulei Shang, and Jun Gao \\ Department of Geophysics, Yunnan University, 2 North Green Lake Road, Kunming, Yunnan 650091, P. R. China, guangquanli74@gmail.com
}

\begin{abstract}
Mixing-transport of solute entering at sinkholes or from within the limestone matrix in cavernous conduits is an important process for contaminant migration in karst aquifers. This process may be described with a one-dimensional advection-dispersion equation incorporating the fluxes of solute and water across the conduit wall. For the dilution-dispersion equation, which does not include solute flux across the wall but has the flux of water through the wall, the sufficient and necessary condition for neglecting conduit dispersion is showed by scale analysis to be $L_{P} \gg a$, where $a$ is the conduit radius, and $L_{P}$ is the spatial scale of the solute plume. A straightforward necessary and practically, though not strictly, sufficient condition is $a / W T_{B} \ll 1$, where $W$ is the mean velocity of conduit flow, and $T_{B}$ is the time scale of the breakthrough curve. For the releasing-dispersion equation, which includes the fluxes of water and solute across the wall, $L_{P} \gg a$ is still a sufficient condition, but no longer a necessary one. The inequality $a \ll W T_{B}$ is neither a necessary condition nor a sufficient condition.
\end{abstract}

\section{INTRODUCTION}

The most distinctive feature of karst aquifers is a set of interconnected caves or conduits that form complex underground drainage systems for water and contaminants (Shuster and White, 1971; Kiraly, 1998). These large conduits, often connected to sinkholes and perennial springs at their upstream and downstream ends, respectively, are formed by the dissolving action of acid and aggressive rainwater on carbonate bedrock (usually limestone, dolomite, or marble) over thousands to millions of years. Because the scales of water flow and solute concentration of interest for karst aquifers is typically comparable to, or smaller than, the conduit lengths, sinkholes, springs, and conduits behave like singular points and lines, the prevalence of which makes it a formidable task to accurately model flow and transport in the aquifers.

There are some important works regarding modeling contaminant transport in karst conduits with leaky walls. Tang et al. (1981) developed a model for solute transport in a single fracture, in which the mass transfer between the fracture and the rock matrix is assumed to be diffusive. The weakness of the model is the absence of active solute and water exchange between the fracture and the matrix, which is not realistic for karst aquifers. Contaminant transport in aquifers containing numerous small fractures is often described by a dual-porosity model and a continuum approach, in which contaminant exchange between fractures and porous matrix is modeled by a transfer coefficient representing a diffusive process (Millington and Quirk, 1961; Bear et al., 1993). There are two problems when applying the dual-porosity model to karst aquifers. One is that the exchange of solute between conduits and the matrix is assumed to be diffusive, and the other is that the aforementioned singularity caused by the prevalence of several large conduits was not considered. A numerical model called CAVE (Carbonate Aquifer Void Evolution) developed by Clemens et al. (1996) aims to model the water and calcium exchange between a conduit and the matrix. The liability of CAVE is that the calcium transfer between the matrix and the conduit is diffusion-like (Dreybrodt and Eisenlohr, 2000). In reality, the solute transfer from the matrix to the conduit can be advective or dispersive.

Equations including advection and dispersion were developed to account for retention in immobile-fluid regions (2RNE) and describe transport in a single conduit (Field and Pinsky, 2000; Goldscheider, 2008). The idea is use of the Green's function for the boundary value problem to model solute transport in a conduit. Weaknesses of this analytical model are that advective dilution caused by seepage flow from the matrix is neglected and the conduit dispersion is held constant to facilitate solution. Instead, retention in immobile-fluid regions is used to simulate the tailing phenomena observed in breakthrough curves at springs. Birk et al. (2005) addressed the mixing of matrix water and conduit water and the consequences with respect to interpretation of the spring breakthrough curve. In that scenario, there was no solute flux from the matrix into the conduit. A weakness of their model is the advective dilution induced by the seepage flow from the matrix was not considered.

Water in karst conduits is typically a combination of the water entering at sinkholes and water released from the limestone matrix. In a similar vein, contaminants in karst conduits consist of those entering through sinkholes and those released from the matrix. The mixing-transport of contaminants in a conduit, for the case of negligible conduit dispersion, is analytically solved using the method of characteristics (Li, 2009a; Li, 2009b). The motivation of this article is to investigate when conduit dispersion can be 
neglected for mixing-transport of contaminants in a karst conduit. The results can provide us with insight on when the analytical solution to a simplified equation neglecting dispersion is applicable, as well as when numerical solution of the full equation is needed.

\section{Mixing-Transport of Contaminants in a Conduit}

The transport of solute by flow in a solid-wall pipe can be described with a one-dimensional advection-dispersion equation (Taylor, 1954). Our problem is different in that both water and solute seep into the conduit from the permeable wall. Though complicated, the mixing-transport of contaminants (solute) in a karst conduit may be described using a relatively simple one-dimensional equation. With a consideration of conduit dispersion, solutemass conservation gives ( $\mathrm{Li}$ et al., 2008; Li, 2009a)

$$
\frac{\partial C}{\partial t}+\frac{\partial}{\partial z}\left(W C-D_{c} \frac{\partial C}{\partial z}\right)=\frac{2}{a} J
$$

where $t[\mathrm{~T}]$ is time, $z[\mathrm{~L}]$ is the conduit-wise distance from the sinkhole, $a[\mathrm{~L}]$ is the conduit radius, $C\left[\mathrm{M} \mathrm{L}^{-3}\right]$ is the concentration of solute in the conduit (averaged over the cross-sectional area), $W\left[\mathrm{~L} \mathrm{~T}^{-1}\right]$ is the mean speed of conduit flow, $D_{c}\left[\mathrm{~L}^{2} \mathrm{~T}^{-1}\right]$ is conduit dispersion, and $J[\mathrm{M}$ $\left.\mathrm{L}^{-2} \mathrm{~T}^{-1}\right]$ is the specific solute flux across the conduit wall.

Water mass conservation gives

$$
W(z)=W_{0}+\frac{z}{\tau}
$$

where $W_{0}$ is the mean speed of flow at the sinkhole, and $\tau$ is defined as:

$$
\tau=\frac{a}{2 q},
$$

where $q$ is the specific water flux across the wall.

The conduit dispersion can be parameterized by $(\mathrm{Li}$, 2004; Li et al., 2008)

$$
D_{c}=a W .
$$

This equation for the dispersion coefficient is for turbulent conduit flow, not for laminar flow. The dispersion coefficient for laminar flow is dependent of the square of velocity, often called Taylor dispersion (Bear, 1972).

\section{Scale Analysis of Dilution-Dispersion Equation without Wall Solute Flux}

There is an inhomogenous term on the right side of Equation (1). Also, both the flow velocity $W$ and the conduit dispersion $D_{c}$ are dependent variables. For these reasons, it is difficult to directly evaluate the equation. Two steps are taken to evaluate the equation. The first step is to simplify the equation into an equation that ignores the solute flux but includes the water flux across the wall, which is called the dilution-dispersion equation because clean seepage water dilutes contaminants that have entered at sinkholes. Scale analysis is used decide about the significance of conduit dispersion. Then the conclusion from the first step is used to evaluate whether or not it can apply to the mixing-transport equation incorporating the fluxes of water and solute across the wall, called the (solute) releasing-dispersion equation.

\section{A Sufficient and Necessary Condition for Neglecting Dispersion in \\ Dilution-Dispersion EQuATION}

Ignoring the flux of solute across the conduit wall, Equation (1) simplifies to

$$
\frac{\partial C}{\partial t}+\frac{\partial}{\partial z}(W C)=\frac{\partial}{\partial z}\left(D_{c} \frac{\partial C}{\partial z}\right)
$$

Without losing generality and considering the rising limbs of the breakthrough curves, scale analysis of the above equation yields

$$
\frac{C}{T_{B}}-W \frac{C}{L_{P}}=D_{c} \frac{C}{L_{P}^{2}},
$$

where $T_{B}$ and $L_{P}$ are the time and spatial scales of the solute plume, respectively. Because Equation (6) consists of three terms, it follows that the dispersion term can be neglected when and only when

$$
L_{P}^{2} \gg D_{c} T_{B}
$$

\section{A MORE STRAIGHTFORWARD NECESSARY CONDITION}

In the above section, a sufficient and necessary condition for neglecting conduit dispersion in the dilution-dispersion equation is defined. This subsection aims to derive another, more straightforward sufficient and necessary condition.

If dispersion can be neglected in Equation (6), inequality (7) must be valid as a necessary condition. Substituting Equation (7) into (6) yields:

$$
L_{P} \sim W T_{B},
$$

which states that the transport is advection-dominated. Substituting Equations (4) and (8) into Equation (7) yields

$$
a \ll W T_{B},
$$

which is a necessary condition for neglecting conduit dispersion in Equation (5).

Below is a proof that the inequality in Equation (9) alone is not a sufficient condition for neglecting conduit dispersion. Suppose that with condition Equation (9), the dispersion term in Equation (5) cannot be neglected with respect to the advection term, or more explicitly,

$$
W \frac{C}{L_{P}} \sim<D_{c} \frac{C}{L_{P}^{2}},
$$


where $\sim<$ means being roughly equal to or smaller than. Then

$$
L_{P} \sim<a .
$$

Combining this inequality with Equation (9) yields

$$
W T_{B} \gg a>\sim L_{P},
$$

From the first and last terms of this inequality, the unsteady term, or the first term in Equation (5), can be neglected with respect to the advection term. Thus the transport is nearly steady, and thus we get $L_{P} \sim a$. Inequality (12) becomes

$$
W T_{B} \gg L_{P} \sim a .
$$

If there is a solution allowed under restraint by Equation (13), the condition imposed by Equation (9) will not be a sufficient for neglecting conduit dispersion.

Now we investigate whether or not there is a solution allowed under restraint by Equation (13). As above, the problem is nearly steady, and thus from Equation (5), we have

$$
\frac{\partial}{\partial z}\left(W C-D_{c} \frac{\partial C}{\partial z}\right)=0
$$

Note that the terms in the parentheses are exactly the solute flux in the conduit. Equation (14) means that the solute flux is a constant through the conduit. This equation is a secondorder ordinary differential equation, the solution of which requires two boundary conditions, one at the conduit entrance and the other at the exit. We would let the conduit length roughly equal to the radius, and at the same time, prescribe two constant-concentration conditions for the two ends. Thus, a physically meaningful solution is found that satisfies the restraint imposed by Equation (13). Therefore, condition imposed by Equation (9) is not strictly sufficient for neglecting conduit dispersion.

However, in practical field circumstances, such as dyetracing experiments, the problem becomes better specified. For instance, the breakthrough curves of dye or contaminants at the conduit entrance and the exit, or the boundary conditions at the two ends, are invariably transient or unsteady. Thus the above boundary conditions are not met in field cases, and Equation (14) is typically not satisfied. Therefore, the above supposition that the dispersion term in Equation (5) cannot be neglected with respect to the advection term under the condition imposed by Equation (9), is invalid for field cases. In other words, the condition imposed by Equation (9) is sufficient for neglecting conduit dispersion in practical field cases.

The condition imposed by Equation (9) is not strictly sufficient for neglecting conduit dispersion, because the inequality in Equation (13) may be satisfied. Naturally, a violation of the inequality in Equation (13) may result in a sufficient condition such as

$$
L_{P} \gg a \text {. }
$$

Actually, if the above inequality is satisfied, through Equation (6), the dispersion term must be negligible with respect to the advection term (a sufficient condition). On the other hand, if conduit dispersion can be neglected in Equation (6), the above inequality must be valid (a necessary condition). Therefore, inequality (15) is a necessary and sufficient condition.

\section{AnAlysis For ReleAsing-DisPersion EQuation}

Whether or not inequality Equation (7), namely, $L_{P}^{2} \gg D_{c} T_{B}$, is a condition sufficient or necessary or both for neglecting dispersion in the releasing-dispersion equation, where some solute enters through the conduit wall (Equation (1)), will be investigated in this section. This category is more difficult to analyze in that the right side of the equation is non-zero.

When $L_{P}^{2} \gg D_{c} T_{B}$ is satisfied, the dispersion term in Equation (1) must be negligible. However, it is no longer a necessary condition. Suppose the first term of Equation (1) is roughly equal to or smaller than the third term, and the third term, the dispersion, can be neglected. Then both terms can be ignored, in which case the main balance is between the second term and the right-hand side. That is

$$
W \frac{C}{L_{P}} \sim \frac{2}{a} J .
$$

With the use of $J=C_{m} q$, wherein $C_{m}$ is the concentration of solute released from the matrix, the above equation transforms to $L_{P} \sim a W C / 2 q C_{m}$. It follows that the initial supposition is possible, in which case the spatial scale of the solute plume is described by this equation. Therefore, $L_{P}^{2} \gg D_{c} T_{B}$ is no longer a necessary condition for neglecting conduit dispersion.

We now ask whether or not the inequality in Equation (9), $a \ll W T_{B}$, is a sufficient condition. Following the discussion in the last section, a physical solution can be found, except that the right side of Equation (14) now has a non-homogeneous term resulting from the wall solute flux. Therefore, as before, the inequality in Equation (9) is not a strictly sufficient condition.

In the previous section, we showed that the inequality in Equation (9) is a necessary condition for neglecting conduit dispersion. This is because the main balance in the dilutiondispersion equation is between the first and second terms when dispersion is negligible. However, for the releasingdispersion equation, that is not valid any longer, because the non-homogenous term now complicates the equation. Thus, we can not always get the inequality in Equation (9) as in the last section. Therefore, the inequality in Equation (9) is not a strictly necessary condition for neglecting conduit dispersion in the releasing-dispersion equation. In a quick summary, the inequality in Equation (9) is neither a sufficient nor a necessary condition. 
Table 1. The parameters of the designed numerical example.

\begin{tabular}{|c|c|c|}
\hline Parameter & Value & Units \\
\hline Conduit radius, $a$ & 2.0 & $\mathrm{~m}$ \\
\hline Conduit length, $L$ & 100.0 & $\mathrm{~m}$ \\
\hline Matrix solute concentration, $C_{m}$ & 30.0 & $\mathrm{mg} \mathrm{L}^{-1}$ \\
\hline Water flux at sinkhole, $Q_{0}$ & 0.18 & $\mathrm{~m}^{3} \mathrm{~s}^{-1}$ \\
\hline Water flux from conduit wall, $Q_{r}$ & 1.62 & $\mathrm{~m}^{3} \mathrm{~s}^{-1}$ \\
\hline Total water flux at spring, $Q_{s}$ & 1.8 & $\mathrm{~m}^{3} \mathrm{~s}^{-1}$ \\
\hline Specific water flux across wall, $q$ & 0.00129 & $\mathrm{~m} \mathrm{~s}^{-1}$ \\
\hline Reynolds number ${ }^{\mathrm{a}}, \operatorname{Re}($ cell) & 0.028 & \\
\hline
\end{tabular}

${ }^{a}$ The Reynolds number controls the accuracy of spatial discretization in the numerical solution.

At the beginning of this section, $L_{P}^{2} \gg D_{c} T_{B}$ is shown to be a sufficient but not a necessary condition for neglecting conduit dispersion in the releasing-dispersion equation. Similarly, condition $L_{P} \gg a$ is not a necessary condition. Supposing $L_{P} \sim<a$ and following the same procedure, the second term in Equation (1) can be roughly equal to or smaller than the third term, while the third term can be neglected. Thus, both the second and third terms can be ignored in the equation. In this case, the main balance is between the first and last terms, and the time-scale of the breakthrough curve is determined by a similar equation, $T_{B} \sim a C / 2 q C_{m}$. Therefore, $L_{P} \gg a$ is no longer a necessary condition for neglecting conduit dispersion. Of course, this equality is a sufficient condition, because the dispersion term can be neglected with respect to the advection term, and therefore, can be neglected from Equation (1).

\section{A Numerical ExAmple}

In the above two sections, through scale analysis, we investigate whether or not three inequalities, $L_{P}^{2} \gg D_{c} T_{B}$, $W T_{B} \gg a$, and $L_{P} \gg a$, are sufficient and/or necessary

Break through Curve at Conduit Exit, ts $=0.1$ hour

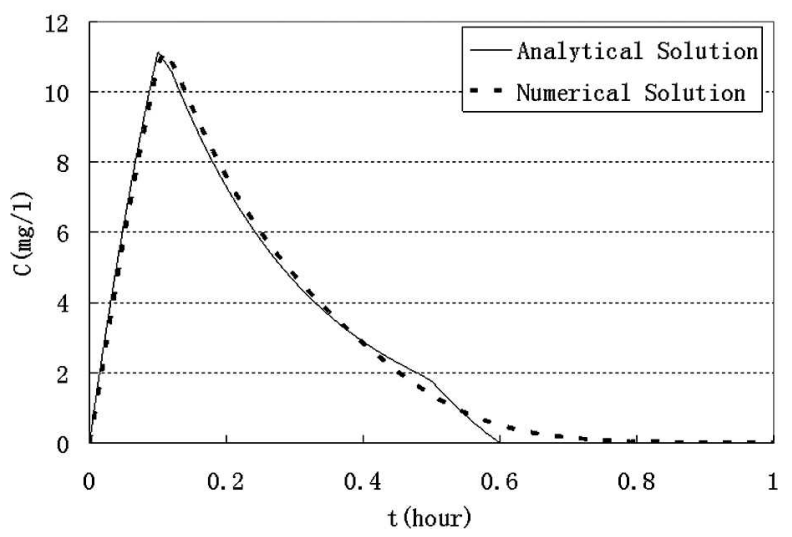

Figure 1. Comparison between the breakthrough curves from the numerical solution of the releasing-dispersion equation and the analytical solution, in which the duration of release $t_{s}=0.1$ hour.

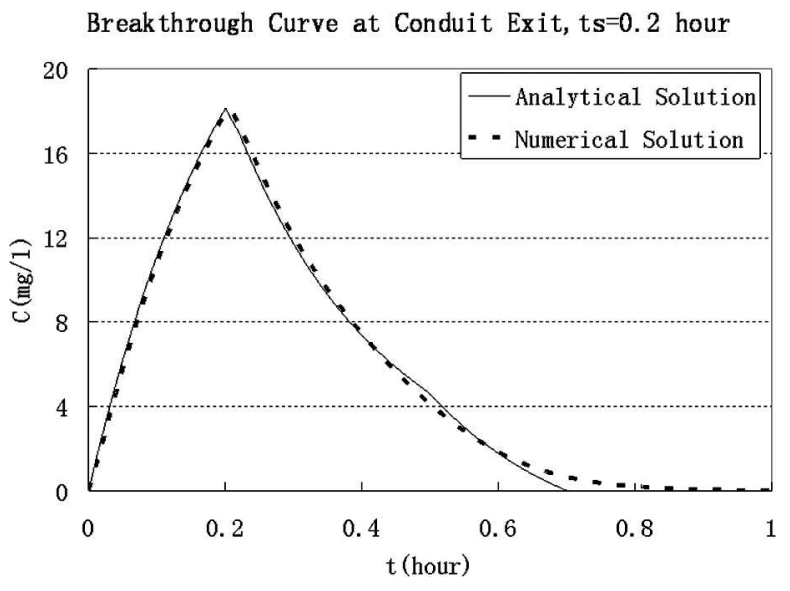

Figure 2. Same as Figure 1, except that $t_{s}=0.2$ hour.

conditions. In this section, a numerical method is used to verify that $L_{P} \gg a$ is a sufficient condition for neglecting conduit dispersion in the releasing-dispersion equation.

In the numerical example, solute and water are released from the matrix into a conduit, but no solute enters via a sinkhole. The analytical solution of this problem is presented as online supplementary documents to $\mathrm{Li}$ (2009a), while the numerical solution is sought by the implicit and stable Crank-Nicolson scheme (Li, 2004). Table 1 lists the parameters of the defined example, in which the cell Reynolds number is very small, which guarantees the accuracy of the numerical solution. Matches between the numerical solution and the analytical solution are plotted in Figures 1 through 5. The durations of release $t_{s}$ in Figures 1 through 5 are $0.1,0.2,0.5,2.0$, and 6.0 hours, respectively. Because inequality $L_{P} \gg a$ is satisfied, the matches between the numerical solutions and the analytical solutions appear to be good. This numerical result verifies that the inequality $L_{P} \gg a$ is a sufficient condition, as showed by the preceding scale analysis.

Break through Curve at Conduit Exit, ts $=0.5$ hour

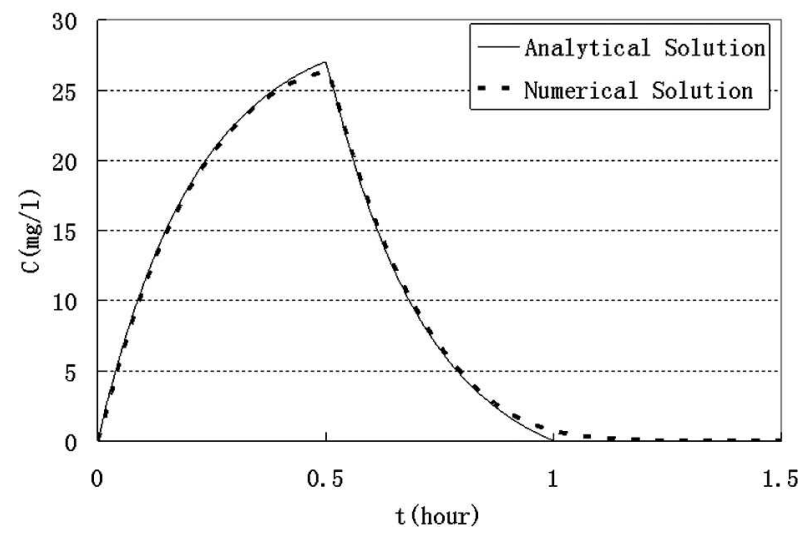

Figure 3. Same as Figure 1, except that $t_{s}=0.5$ hour.

Journal of Cave and Karst Studies, December 2010•153 
Break through Curve at Conduit Exit, ts $=2.0$ hour

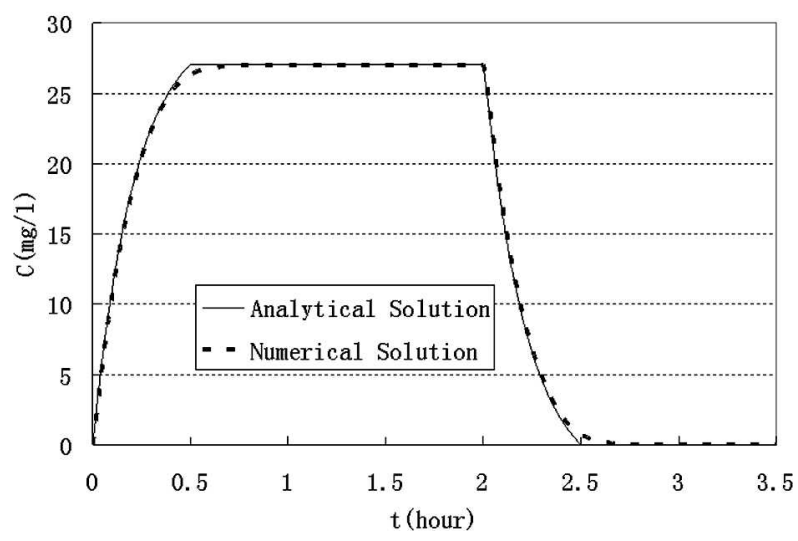

Figure 4. Same as Figure 1, except that $t_{s}=2.0$ hours.

\section{Discussion}

The results of our scale analysis of the significance of dispersion in mixing-transport in karst conduits are summarized in Table 2. For the dilution-dispersion equation that includes flux of water but not solute from the wall, a straightforward necessary and practically sufficient condition for neglecting conduit dispersion is $W T_{B} \gg a$. A potential application of this condition is the case where no contaminants are flowing from the matrix and the released matrix water dilutes contaminants entering via sinkholes, i.e., dye-tracing experiments.

Suppose that a dye-tracing experiment has a mean flow velocity $\left(W_{0}\right)$ of $0.1 \mathrm{~m} \mathrm{~s}^{-1}$ and a conduit has a radius $a$ of $1 \mathrm{~m}$. In order to neglect conduit dispersion, a ratio of $a / W T_{B}=0.01$ would require dye release to have a duration satisfying $T_{B}>100 a / W=1000$ seconds. In other words, a dye trace with a release duration of tens of minutes would result in negligible conduit dispersion, and the dilutiontransport model developed in $\mathrm{Li}$ (2009b) should be applicable. If applying that model yields an unreasonable overestimation of the conduit length and spring water flux, we would conclude that the drainage system most likely consists of a network of conduits, rather than a single conduit, or there is a strong interaction between conduit and matrix. Notably, vortices at conduit enlargements could contribute to greater dispersion than usual. To exclude that possibility, a dye-release duration up to several hours would be desirable to neglect conduit dispersion. Such a requirement is seldom satisfied when implementing dye-tracing experiments. Nevertheless, a long duration induced by rain events may mimic the suggested dye-input rate.

When there is solute flux being released from the matrix, things become more difficult to analyze because the inhomogenous term, i.e., the solute flux across the wall, complicates the releasing-dispersion equation so that scale analysis is more difficult to conduct. However, we nonetheless get the results listed in Table 2. A notable
Break through Curve at Conduit Exit, ts $=6.0$ hour

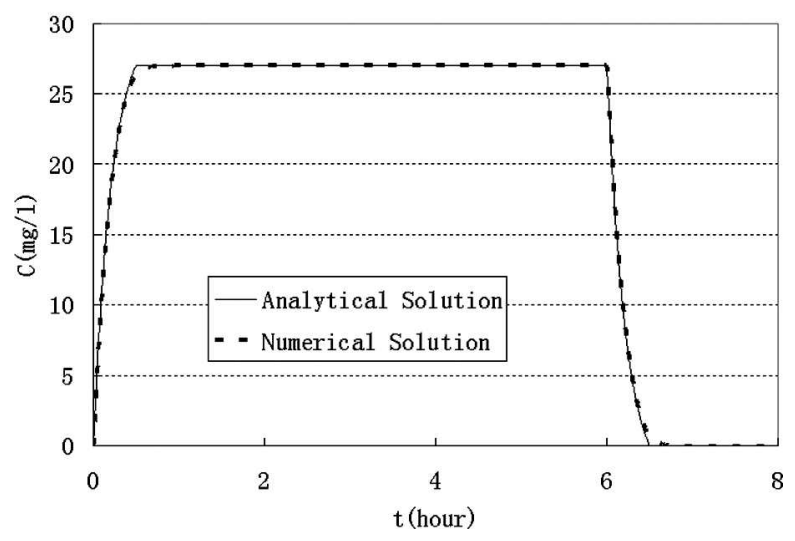

Figure 5. Same as Figure 1, except that $t_{s}=6.0$ hours.

feature of the numerical solutions in Figures 1 through 5 is the long tail in the numerical solution, which is believed to result from variable conduit dispersion. Nevertheless, the tail caused by variable conduit dispersion is much smaller than that observed in field experiments; see Birk et al. (2005). This illustrates that retention in immobile-water region is a more important mechanism to produce tailing.

\section{Conclusions}

The mixing-transport of contaminants in a karst conduit can be described using a one-dimensional advection-dispersion equation incorporating fluxes of water and solute across the conduit wall. We call the equation without solute flux but with water flux across the wall the dilution-dispersion equation, while calling the equation with fluxes of water and solute across the wall the releasing-dispersion equation.

Scale analysis was performed to investigate the significance of dispersion in the dilution-dispersion equation. As dye-tracing experiments may be described with the equation, the result of our scale analysis has important applications to dye tracing experiments and similar physical processes, such as surface contaminants being carried into a sinkhole and transported in a conduit. A rule of thumb is that when and only when $a / W T_{B} \ll 1$, conduit dispersion can be ignored. This inequality, in conjunction with the dilution-transport model ( $\mathrm{Li}, 2009 \mathrm{~b})$, provides us with a tool to investigate whether the drainage system under dye-tracing experiments mainly consists of a single conduit or a conduit network. More specifically, if applying the model results in an unreasonable estimate of conduit parameters such as the length, the conduit system is likely to consist of a network, or there is a strong interaction between conduit and matrix.

When contaminants sequestered in the rock matrix are released into the conduit, the process may be described with the releasing-dispersion equation. In this case, our scale analysis reveals that $L_{P} \gg a$ is a sufficient but not 
Table 2. Results from our scale analysis of the significance of dispersion in mixing-transport in a karst conduit. Note that $W T_{B} \gg a$ is a practically sufficient condition for neglecting conduit dispersion in the dilution-dispersion equation.

\begin{tabular}{lcl}
\hline Condition & $\begin{array}{c}\text { Conduit Dispersion Neglected in } \\
\text { Dilution-Dispersion Equation }\end{array}$ & $\begin{array}{c}\text { Conduit Dispersion Neglected in } \\
\text { Releasing-Dispersion Equation }\end{array}$ \\
\hline$L_{P}^{2} \gg D_{C} T_{B}$ & Sufficient and Necessary & Sufficient and Unnecessary \\
$W T_{B} \gg a$ & Necessary but not Sufficient & Neither Necessary nor Sufficient \\
$L_{P} \gg a$ & Sufficient and Necessary & Sufficient and Unnecessary \\
\hline
\end{tabular}

necessary condition for neglecting conduit dispersion, which is verified by a preliminary numerical study. In reality, solute transport in karst conduits is a result of different interactive processes. If conduit dispersion can be shown to be negligible, we may be able to detect other mechanisms, such as retention in immobile water and multiple pathways, dominating the mixing-transport, thus being able to resolve different mechanisms. This is the primary aim of our study.

\section{ACKNOWLEDGEMENTS}

Financial support of this research was provided by the University Fund of Yunnan University under contract KL090020. The authors are deeply grateful to the Editor Malcolm S. Field, the Associate Editor Gregory S. Springer (who edited the paper for English usage), and two anonymous reviewers for their insightful comments and constructive suggestions.

\section{REFERENCES}

Bear, J., 1972, Dynamics of Fluids in Porous Media, New York, Dover, $764 \mathrm{p}$.

Bear, J., Tsang, C-F., and de Marsily, G., 1993, Flow and Contaminant Transport in Fractured Rock, San Diego, Academic Press, 560 p.

Birk, S., Geyer, T., Liedl, R., and Sauter, M., 2005, Processed-based interpretation of tracer tests in carbonate aquifers: Ground Water, v. 43 , no. 3 , p. $381-388$.

Clemens, T., Huckinghaus, D., Sauter, M., Liedl, R., and Teutsch, G., 1996, A combined continuum and discrete network reactive transport model for the simulation of karst development, in Calibration and
Reliability in Groundwater Modeling: Proceedings of the ModelCARE 96 Conference: International Association of Hydrological Sciences Publ. 237, p. 309-318.

Dreybrodt, W., and Eisenlohr, L., 2000, Limestone dissolution rates in karst environments, in Klimchouk, A., Ford, D., Palmer, A., and Dreybrodt, W., eds., Speleogenesis, Evolution of Karst Aquifers, Huntsville, Ala., National Speleological Society, p. 136-148.

Field, M.S., and Pinsky, P.F., 2000, A two-region nonequilibrium model for solute transport in solution conduits in karstic aquifers: Journal of Contaminant Hydrology, v. 44, no. 3-4, p. 329-351.

Goldscheider, N., 2008, A new quantitative interpretation of the long-tail and plateau-like breakthrough curves from tracer tests in the artesian karst aquifer of Stuttgart, Germany: Hydrogeology Journal, v. 16, p. 1311-1317.

Kiraly, L., 1998, Modeling karst aquifers by the combined discrete channel and continuum approach: Bulletin du. Centre d'Hydrogeologie, v. 16 , p. $77-98$.

Li, G., 2004, Laboratory Simulation of Solute Transport and Retention in a Karst Aquifer [Ph. D. thesis], Tallahassee, Florida State University, $194 \mathrm{p}$.

Li, G., Loper, D.E., and Kung, R., 2008, Contaminant sequestration in karstic aquifers: Experiments and quantification: Water Resources Research, v. 44, W02429 p. doi:10.1029/2006WR005797.

Li, G., 2009a, Analytical solution of advective mixing in a conduit: Ground Water, v. 47, no. 5, p. 714-722. doi:10.1111/j.1745-6584.2009. 00575.x.

Li, G., 2009b, Analytical solution of advective dilution of conduit flow. in preparation.

Millington, R.J., and Quirk, J.M., 1961, Permeability of porous solids: Faraday Transactions, v. 57, p. 1200-1207.

Shuster, E., and White, W., 1971, Seasonal fluctuations in the chemistry of limestone springs: A possible means for characterizing carbonate aquifers: Journal of Hydrology, v. 14, p. 93-128.

Tang, D.H., Frind, E.O., and Sudicky, E.A., 1981, Contaminant transport in fractured porous media: Analytical solution for a single fracture: Water Resources Research, v. 17, p. 555-564.

Taylor, G.I., 1954, The dispersion of matter in turbulent flow through a pipe: Proceedings of the Royal Society (London) A, v. 223, p. 446-468. doi:10.1098/rspa.1954.0130. 